\title{
Oficina musical participativa para o Bem-Estar Subjetivo e Psicológico de usuários em internação psiquiátrica
}

\author{
Participatory music workshop for the Subjective and Psychological Well-being of users in psychiatric
} hospitalization

Taller musical participativo para el Bienestar Subjetivo y Psicológico de usuarios en hospitalización psiquiátrica

1. Universidade Estadual Paulista "Júlio de Mesquita Filho", Faculdade de Medicina de Botucatu, Departamento de Enfermagem. Botucatu, SP, Brasil.

2. Universidade Estadual Paulista "Júlio de Mesquita Filho", Faculdade de Medicina de Botucatu, Departamento de Enfermagem, Programa de Residência Multiprofissional em Saúde Mental. Botucatu, SP, Brasil.

3. Universidade Estadual Paulista "Júlio de Mesquita Filho", Faculdade de Medicina de Botucatu, Departamento de Enfermagem, Programa de Residência Multiprofissional em Saúde da Família. Botucatu, SP, Brasil.

4. Universidade Federal de São Paulo, Escola Paulista de Enfermagem, Departamento de Enfermagem Clínica e Cirúrgica. São Paulo, SP, Brasil.
Autor correspondente:

Eduardo Gabriel Cassola

E-mail: eduducassola@gmail.com

Recebido em 06/04/2021.

Aprovado em 23/07/2021.

\section{Resumo}

Objetivo: identificar as contribuições de uma intervenção musical participativa sobre o Bem-Estar Psicológico e Subjetivo na assistência da enfermagem de usuários com transtorno mental grave e persistente durante a internação. Método: pesquisa quase-experimental, do tipo antes e depois, realizada em uma instituição hospitalar do estado de São Paulo. Dez usuários em internação psiquiátrica participaram das quatro oficinas musicais. O repertório atendeu às suas preferências e foram distribuídos instrumentos musicais para promover a participação. Aplicou-se a escala de Bem-Estar Psicológico e dos Afetos Positivos e Negativos ao início e ao término de cada oficina. Os dados foram analisados considerando Intervalo de Confiança de $95 \%$. Resultados: não foram identificadas diferenças estatísticas entre os valores encontrados antes e depois das oficinas musicais para as variáveis do Bem-Estar e Afetos Positivos e Negativos. Os desfechos do Bem-Estar Subjetivo foram mais lineares quando comparados ao Bem-Estar Psicológico, observando-se aumento nos afetos positivos e diminuição dos afetos negativos. Conclusão e implicações para a prática: a oficina musical participativa favorece o bem-estar subjetivo de usuários com transtornos mentais graves e persistentes. Essa intervenção correspondeu a uma tecnologia leve potencial para a enfermagem atuar de modo autônomo e qualificado no cuidado psicoemocional do usuário na internação psiquiátrica.

Descritores: Enfermagem Psiquiátrica; Música; Saúde Mental; Terapias Complementares; Transtornos Mentais.

\section{Abstract}

Objective: to identify the contributions of a participatory musical intervention on Psychological and Subjective Well-Being in nursing care for users with severe and persistent mental disorder during hospitalization. Method: this is a quasi-experimental before-and-after research, carried out in a hospital in the state of São Paulo. Ten users in psychiatric hospitalization participated in the four music workshops. The repertoire catered to their preferences and musical instruments were distributed to promote participation. Psychological Well-Being and the Positive and Negative Affect Schedule were applied at the beginning and at the end of each workshop. Data were analyzed considering a confidence interval of $95 \%$. Results: no statistical differences were identified between the values found before and after the musical workshops for the variables Well-Being and Positive and Negative Affects. The outcomes of Subjective Well-Being behaved more linear when compared to Psychological Well-Being, with an increase in positive affects and a decrease in negative affects. Conclusion and implications for practice: participatory music workshops favor the subjective well-being of users with severe and persistent mental disorders. This intervention corresponded to a potentia light technology for nursing to act autonomously and qualified in the psycho-emotional care of users in psychiatric hospitalization.

Keywords: Psychiatric Nursing; Music; Mental Health; Complementary Therapies; Mental Disorders.

\section{Resumen}

Objetivo: identificar los aportes de una intervención musical participativa sobre el Bienestar Psicológico y Afectos Positivos y Negativos en el cuidado de enfermería de usuarios con trastorno mental severo y persistente durante la hospitalización. Método: investigación cuasi experimental, del tipo antes y después, realizada en un hospital del estado de São Paulo. Diez usuarios en hospitalización psiquiátrica participaron en los cuatro talleres de música. El repertorio cumplió con sus preferencias y se distribuyeron instrumentos musicales para promover la participación. La escala de Bienestar Psicológico y Afectos Positivos y Negativos se aplicó al inicio y al final de cada taller. Los datos se analizaron considerando un intervalo de confianza del $95 \%$. Resultados: no se identificaron diferencias estadísticas entre los valores encontrados antes y después de los talleres musicales para las variables de bienestar y afectos positivos y negativos. Los resultados del Bienestar Subjetivo se comportaron de forma más lineal en comparación con el Bienestar Psicológico, con un aumento de los afectos positivos y una disminución de los afectos negativos. Conclusión e implicaciones para la práctica: el taller de música participativa favorece el bienestar subjetivo de los usuarios con trastornos mentales graves y persistentes. Esta intervención correspondió a una potencial tecnología de luz para que Enfermería actuara de manera autónoma y calificada en la atención psicoemocional del usuario durante la hospitalización psiquiátrica.

Descriptores: Enfermería Psiquiátrica; Música; Salud Mental; Terapias Complementarias; Transtornos Mentales. 


\section{INTRODUÇÃO}

As terapias complementares envolvem abordagens, práticas e saberes que buscam estimular, por meio da aplicação de tecnologias seguras e eficazes, os mecanismos naturais de prevenção de agravos e de recuperação da saúde, recebendo essa classificação quando integradas à racionalidade biomédica'. No campo da saúde mental, vem se manifestando como oposição ao modelo manicomial, ainda enraizado no campo da assistência. Dentre as práticas complementares, este estudo priorizou a música no contexto da atenção hospitalar, por representar um modo de expressão intrínseco ao humano que possibilita partilhar afetos e emoções, fortalecendo as interações e relações humanas ${ }^{2}$.

O emprego da música, além de repercutir positivamente sobre os mecanismos biológicos, atua sobre outras esferas que permitem a potencialização da qualidade de vida, a melhora das condições físicas, sociais, emocionais, espirituais, bem como compreende uma forma para a expressão do sujeito. Sendo um recurso não farmacológico, essa tecnologia proporciona diversos benefícios e se caracteriza como uma terapia com favorável custo-efetividade ${ }^{3-5}$.

O conjunto de atividades motoras e cognitivas envolvidas no processamento da música é chamado de função cerebral. Para que ocorra a interpretação musical e melódica, esta função necessita de uma formação complexa de operações mentais, como interpretação dos timbres, harmonias, ritmos, processos cognitivos e emocionais. Para além disto, a música é capaz de incitar a memória não verbal por intermédio das áreas associativas secundárias. Essas permitem acesso ao sistema de percepções integradas, responsáveis pela união de diversas sensações - olfatória, propriocepção, gustativa e visual. Essa integração provoca, a exemplo, lembrança de uma imagem após ouvir determinado som ${ }^{6}$.

Os ritmos biológicos são influenciados pelas manifestações rítmicas que ocorrem no próprio ambiente. Esses ritmos biológicos são ditados pelos "relógios" naturais do cérebro, representados pelo epitálamo e o hipotálamo, respectivamente, responsáveis pelos ritmos circanuais e ritmos circadianos, coordenando as atividades temporais endógenas. O ambiente provoca oscilações constantes, fazendo com que uma espécie necessite variar de um modo o qual aconteça uma adaptação temporal, ou seja, sobrevém a harmonização entre a ritmicidade biológica e os ciclos ambientais ${ }^{6}$.

A música contribui para o desenvolvimento de conteúdos subjetivos do usuário com transtorno mental grave e persistente, produzindo ressignificações, recuperando a autoconfiança, autoimagem e criatividade ${ }^{7}$. Os referenciais teóricos mais relevantes sobre o bem-estar se dão em duas concepções: o Bem-Estar Psicológico (BEP), que investiga o potencial humano e o Bem-Estar Subjetivo (BES), o qual se refere ao estado subjetivo de felicidade ${ }^{2,8}$.

O BES consiste em avaliações de satisfação do sujeito com sua vida e no balanço entre os afetos negativos e positivos, as quais demonstram com que frequência eventos alegres ou angustiantes são vivenciados. Sujeitos com altos escores de afetos negativos se percebem desanimados, tristes e preocupados, enquanto aqueles com elevados escores de afetos positivos percebem-se alegres, confiantes e entusiasmados ${ }^{9}$.

Para que se considere adequado o nível do BES, o sujeito deve reconhecer a manutenção de nível elevado de suas satisfações com a vida, apresentando maior regularidade nas experiências emocionais positivas e menor frequência de experiências negativas. A obtenção desses dados necessita de autoavaliação, que somente pode ser relatada e observada por ele próprio 8 ,10.

Em relação ao $\mathrm{BEP}$, a literatura científica o relaciona à capacidade do sujeito para encarar os desafios da vida, atrelando a formulação psicológica ao desenvolvimento humano, como padrão de emprego, nível de escolaridade, urbanização, entre outros. Essa categoria de bem-estar possui um construto configurado por seis elementos: relacionamento positivo com outras pessoas, autoaceitação, autonomia, domínio do ambiente, crescimento pessoal e propósito de vida ${ }^{8}$.

No ano de 2006, a Política Nacional de Práticas Integrativas e Complementares foi instituída junto ao SUS (Sistema Único de Saúde) ${ }^{11}$. A partir dessa data, houve duas ampliações com a inclusão de novos recursos terapêuticos e, dentre eles, a musicoterapia ${ }^{12-14}$. Essa técnica se caracteriza por utilizar a música e seus elementos como uma intervenção de cuidado, almejando a melhor qualidade de vida para o sujeito ${ }^{15}$.

Visando à atuação dos profissionais de enfermagem, a Resolução 581/2018 do Conselho Federal de Enfermagem garante e reconhece as Práticas Integrativas e Complementares (PIC) como especialidade profissional ${ }^{16}$. Tanto a enfermagem quanto as PIC concentram suas ações no sujeito como um todo, buscando considerar, de modo ampliado, o processo de adoecer, não reduzindo a patologia em si. A música aliada à dimensão afetiva do cuidado de enfermagem representa uma ferramenta terapêutica com amplo escopo de atuação, em especial para a prevenção e promoção da saúde mental ${ }^{17,18}$.

O cuidado em saúde mental não envolve apenas o controle de sintomas e a minimização dos riscos de internação, mas compreende também questões sociais, financeiras, emocionais e pessoais, associadas à coexistência com o adoecimento psíquico ${ }^{19}$. Sendo assim, este estudo objetivou identificar as contribuições de uma intervenção musical participativa sobre o BEP e BES na assistência de enfermagem de usuários com transtorno mental grave e persistente durante a internação.

\section{MÉTODO}

Pesquisa quantitativa, delineada por uma intervenção quase-experimental do tipo antes e depois ${ }^{20}$, voltando-se para os desfechos BEP e BES, mais especificamente os Afetos Positivos e Negativos.

O presente estudo foi realizado em uma unidade de internação psiquiátrica vinculada a um Centro de Atenção Integral à Saúde no interior do estado de São Paulo. A estrutura é composta por 26 leitos distribuídos, segundo a demanda, entre as enfermarias masculina e feminina. A equipe é composta por enfermeiros, 
terapeutas ocupacionais, assistentes sociais, psicólogos, auxiliares administrativos e de enfermagem e médicos clínicos e psiquiatras.

Para a constituição da amostra, foram elencados como critérios de inclusão: maioridade, estar no período de internação, participação nos quatro encontros planejados para a oficina musical. Comprometimento clínico e psiquiátrico que impedia a participação nas oficinas e preenchimento dos instrumentos de coleta de dados foi considerado critério único de exclusão. Desse modo, foram realizados quatro encontros durante a primeira semana de novembro de 2019. Havia 30 usuários em período de internação. Após convite pessoal, 24 aceitaram participar das oficinas. Desses, dois participantes foram excluídos: um por não conseguir responder aos questionários de coleta de dados e outro por não possuir o consentimento do familiar responsável. Mais 11 participantes foram excluídos; desses, dez por desistência e um participante por não responder a segunda aplicação do questionário. Portanto, a intervenção foi finalizada com dez participantes.

O primeiro contato aconteceu nas enfermarias, com intermédio das equipes de referência das enfermarias feminina e masculina. Nessa ocasião, ocorreu apresentação e convite para participação na oficina de música. $O$ familiar e o participante da pesquisa assinaram, respectivamente, o Termo de Consentimento Livre e Esclarecido e o Termo de Assentimento Livre e Esclarecido.

A oficina musical participativa foi constituída por quatro encontros distribuídos em duas semanas com dois encontros semanais, com duração de 45 minutos. A experiência foi conduzida pelos quatro primeiros autores da pesquisa em uma sala reservada em um espaço fora das enfermarias. O planejamento de cada uma das oficinas consistiu em: (1) recepcionar cada um dos dez participantes; (2) explicar sobre a oficina, esclarecendo seu funcionamento e função; (3) aplicar os instrumentos de coleta de dados; (4) apresentar e distribuir os instrumentos musicais de acordo com a predileção dos participantes; (5) estimular a escolha das músicas e a participação de cada durante a execução das canções; (6) aplicar os instrumentos de coleta de dados; (7) encerrar e despedir. Os pesquisadores distribuíram-se entre as atividades, repetindo as mesmas atribuições nos quatro encontros.

Antes de iniciar as atividades da oficina musical, os instrumentos de coleta de dados foram distribuídos e respondidos pelos participantes. Após essa etapa introdutória, foram distribuídos instrumentos de percussão, como pandeiro, triângulo, meialua, chocalho, os quais os participantes puderam escolher o instrumento de preferência. O repertório musical foi constituído de músicas selecionadas pelos participantes como estratégia de integração à oficina. Durante a execução musical, a atuação dos pesquisadores se caracterizou por incentivar os participantes a cantarem e a tocarem; quando necessário, auxiliavam cantando junto dos participantes. Os principais ritmos selecionados foram Baião, Gospel, Rock e Bossa Nova.

Em relação aos instrumentos de coleta de dados, aplicou-se um instrumento de levantamento de dados sociodemográficos e clínico, respondido pelo participante com apoio do respectivo familiar e de informações contidas no prontuário médico. Ao início e término de cada um dos quatro encontros musicais, foram aplicados os instrumentos referentes aos desfechos do BES e do BEP, ambos validados para o Brasil. As observações espontâneas realizadas pelos profissionais da unidade de internação e pelos próprios usuários e o comportamento dos participantes durante as oficinas foram registradas pelos pesquisadores com a finalidade de verificar o comportamento emocional dos participantes.

No que se refere ao BES, foi utilizada a Escala de Afetos Positivos e Negativos. Trata-se de um instrumento autoaplicável, composto por dez adjetivos que representam estados afetivos positivos e dez adjetivos que representam estados afetivos negativos, cujas respostas são organizadas segundo uma escala Likert de cinco pontos. Tanto para os estados afetivos positivos e negativos, pontuações mais altas indicam estados afetivos mais intensos ${ }^{21}$.

A Escala de BES, por sua vez, é constituída de 36 itens distribuídos em seis dimensões (autoaceitação, relacionamento positivo com os outros, autonomia, domínio ambiental, propósito de vida e crescimento pessoal), sendo que cada item pode ser respondido segundo uma escala Likert de seis pontos. Escores mais altos correspondem a maiores índices de BEP, podendo atingir, na totalidade da escala, 216 pontos $^{22}$.

Inicialmente, foi realizado um teste de normalidade para verificar a distribuição dos dados dos domínios e do escore dos instrumentos de coleta. Para os dados que apresentaram uma distribuição simétrica, foi ajustado um modelo misto em medidas repetidas seguidas de Tukey para as comparações múltiplas, para analisar os desfechos nos momentos antes e depois da intervenção em seus quatro momentos. Para aqueles que apresentaram uma distribuição assimétrica, foi ajustado um modelo linear generalizado em medidas repetidas, com distribuição gama, seguido de comparações múltiplas. Considerou-se $p<0,05$ como nível de significância. $O$ programa utilizado para realizar as análises foi o programa SAS, versão 9.4.

De acordo com a Resolução 466/2012 23 , a pesquisa fol apreciada pelo Comitê de Ética em Pesquisa da Faculdade de Medicina de Botucatu, recebendo parecer favorável 3.634.420.

\section{RESULTADOS}

Entre a população do estudo, houve discreta predominância para o sexo feminino, sem parceiros(as), com nível fundamental e sem filhos. A média etária entre o sexo feminino foi de 41,8 anos (19-60 anos), enquanto a população masculina foi de 29,5 (1850 anos). Entre as características clínicas, os participantes receberam diagnóstico relacionados aos transtornos de pensamento e transtornos de humor, sendo que a totalidade fazia uso de antipsicóticos. O tempo médio de internação é de 30 dias. Identificou-se que $40 \%$ da amostra fazia uso de três classes de psicofármacos. A Tabela 1 ilustra a distribuição dos participantes.

A Tabela 2 apresenta os resultados obtidos entre os quatro encontros da oficina musical, em função das seis dimensões e do escore geral da escala de BEP. Detalhadamente, pode-se 
Tabela 1. Caracterização sociodemográficas e clínicas dos participantes. Botucatu, SP, Brasil, 2019

\begin{tabular}{|c|c|c|}
\hline \multirow{2}{*}{ Variáveis } & \multicolumn{2}{|c|}{ Participantes } \\
\hline & $\mathrm{n}$ & $\%$ \\
\hline \multicolumn{3}{|l|}{ Sexo } \\
\hline Masculino & 4 & 40 \\
\hline Feminino & 6 & 60 \\
\hline \multicolumn{3}{|l|}{ Faixa etária } \\
\hline $18-31$ anos & 4 & 40 \\
\hline $32-45$ anos & 2 & 20 \\
\hline $46-60$ anos & 4 & 40 \\
\hline \multicolumn{3}{|l|}{ Estado civil } \\
\hline Sem parceiro(a) & 8 & 80 \\
\hline Com parceiro(a) & 2 & 20 \\
\hline \multicolumn{3}{|l|}{ Nível de instrução } \\
\hline Ensino fundamental completo & 1 & 10 \\
\hline Ensino fundamental incompleto & 5 & 50 \\
\hline Ensino médico completo & 3 & 30 \\
\hline Ensino superior incompleto & 1 & 10 \\
\hline \multicolumn{3}{|l|}{ Filhos } \\
\hline Sem filhos & 6 & 60 \\
\hline $1-3$ & 4 & 40 \\
\hline \multicolumn{3}{|l|}{ Diagnóstico médico (CID-10) } \\
\hline F20 & 5 & 50 \\
\hline F30 & 5 & 50 \\
\hline \multicolumn{3}{|l|}{ Transtorno mental na família } \\
\hline Sim & 2 & 20 \\
\hline Não & 8 & 80 \\
\hline \multicolumn{3}{|l|}{ Etilista } \\
\hline Sim & 1 & 10 \\
\hline Não & 9 & 90 \\
\hline \multicolumn{3}{|l|}{ Drogas ilícitas } \\
\hline Sim & 1 & 10 \\
\hline Não & 9 & 90 \\
\hline \multicolumn{3}{|l|}{ Antipsicóticos } \\
\hline Não & 0 & 0 \\
\hline Sim & 10 & 100 \\
\hline \multicolumn{3}{|l|}{ Antidepressivos } \\
\hline Não & 4 & 40 \\
\hline Sim & 6 & 60 \\
\hline \multicolumn{3}{|l|}{ Benzodiazepínicos } \\
\hline Não & 1 & 10 \\
\hline Sim & 9 & 90 \\
\hline \multicolumn{3}{|l|}{ Anticonvulsivantes } \\
\hline Não & 6 & 60 \\
\hline Sim & 4 & 40 \\
\hline
\end{tabular}

Fonte: dados da pesquisa. afirmar que não houve comportamento linear entre cada uma das dimensões, comparando-as aos quatro encontros da oficina musical. Observou-se que não houve diferença estatística entre os momentos antes e depois de cada encontro entre si, bem como na comparação entre os momentos finais de cada oficina.

Os desfechos relacionados aos afetos positivos e negativos são demonstrados também na Tabela 2. Similarmente, não foram observadas diferenças estatísticas entre os momentos antes e depois em cada um dos quatro encontros. O comportamento desses desfechos, contudo, foram mais lineares quando comparados com o BEP e suas dimensões, isto é, em cada um dos encontros da oficina musical participativa, houve aumento na pontuação dos afetos positivos, com concomitante diminuição dos afetos negativos.

\section{DISCUSSÃO}

A intervenção musical participativa não evidenciou favorecer a percepção do BEP entre os usuários com transtornos mentais graves e persistentes no contexto da internação psiquiátrica. Os resultados, contudo, são promissores para a percepção dos afetos positivos e negativos, uma vez que se observou, respectivamente, aumento e diminuição das pontuações dos referidos desfechos.

Devido ao momento em que os participantes se encontravam, crise psíquica e agudização sintomática, observa-se uma fragilidade no acesso às suas dimensões psíquicas. Sob esse aspecto, os resultados não efetivos do BEP após a intervenção musical devem ser discutidos. A crise e o consequente comprometimento cognitivo que os participantes viviam podem ter afetado a compreensão e crítica de si mesmos.

A experiência da crise está sujeita a diversas influências, como aos fatores relacionados às condições sociodemográficas, doença, terapêutica, relação dos profissionais de saúde e ao próprio usuário com transtorno mental grave e persistente. Nesses momentos, a crise é acompanhada por perdas e limitações nas várias dimensões da vida cotidiana, podendo ser perdas afetivas e cognitivas, limitações na esfera dos estudos, trabalho e lazer ${ }^{24}$.

O comprometimento da cognição abrange diversos aspectos, como questões sociais, memória, atenção, velocidade de processamento e raciocínio, compreensão, fluxo e associação do pensamento e variações de julgamentos ${ }^{25}$.

Por outro lado, no que diz respeito aos resultados dos afetos negativos e positivos, obtidos por meio da intervenção, os dados demonstraram que a música favorece o aumento da pontuação nos afetos positivos e diminuição dos afetos negativos. Como já demonstrado em estudos anteriores, a música pode ser utilizada no processo de restabelecimento da saúde do usuário com transtorno mental grave e persistente, já que ela atua na melhora do BES, do nível funcional geral e das manifestações clínicas ${ }^{3,7}$.

A música é uma forma de linguagem que evidencia o significado emotivo. Primeiro nos toca na esfera afetiva e depois apreendemos seu conteúdo e letra. Na dimensão comunicativa, a música representa um canal que, primeiramente, favorece trocas explorando a linguagem psicobiológica e, em seguida, 


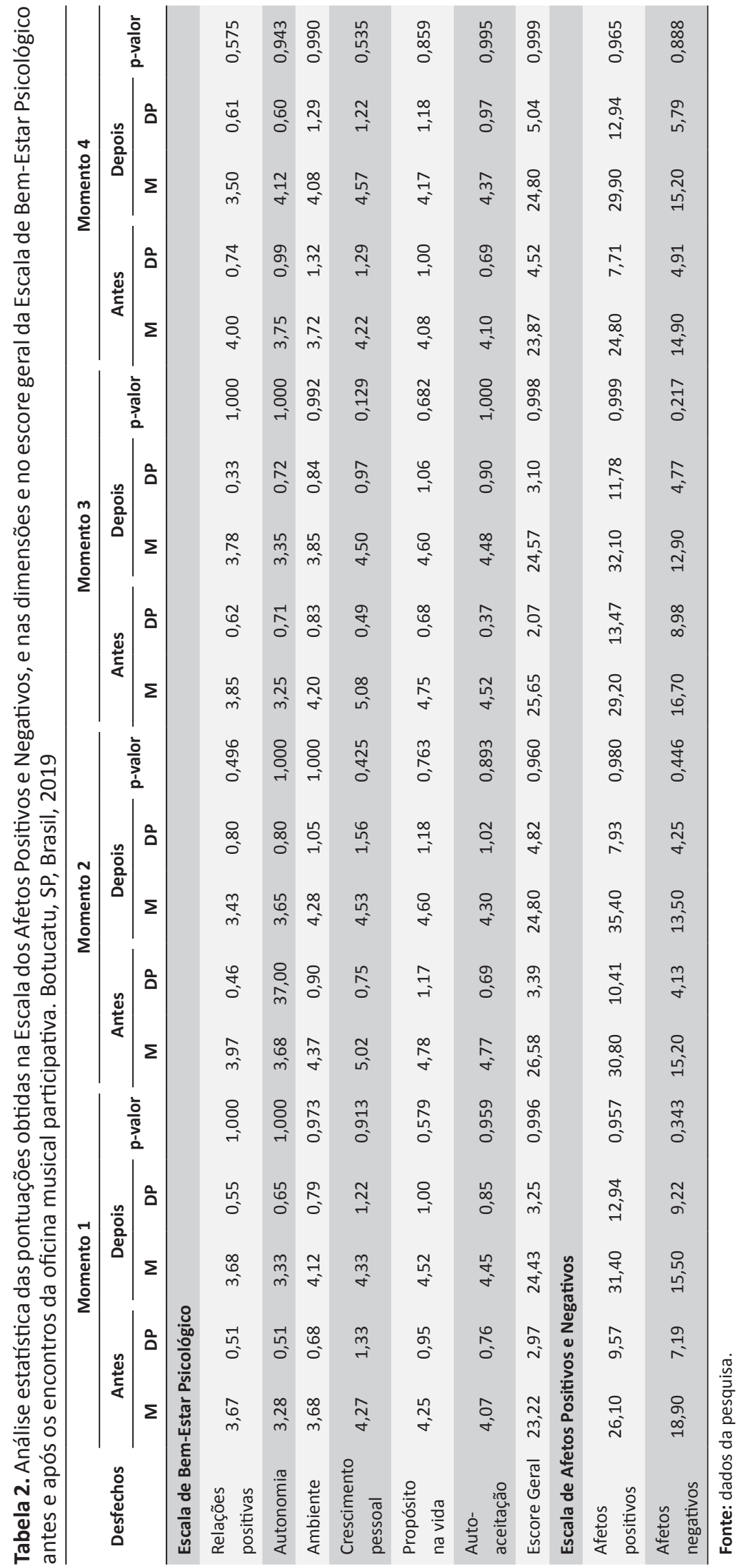


psicolinguística. Quando utilizada intencionalmente, amplia e qualifica os canais comunicativos, favorecendo aspectos relacionais entre enfermeiro e usuário. Ao longo do tempo, a exposição à música favorece que o sujeito tome consciência de quem é e onde está, transformando sua percepção da realidade, promovendo a ampliação de suas trocas com o mundo ${ }^{26,27}$.

A utilização da música auxilia usuários com transtorno mental grave e persistente a adquirir técnicas de modulação emocional por meio de sua personalidade individual, em que, sem esta intervenção, simplesmente acabariam por canalizar suas emoções negativas em outras situações. Assim sendo, a intervenção musical consegue atingir a esfera afetiva do usuário com transtorno mental grave e persistente, possibilitando sua participação e seu engajamento durante a intervenção ${ }^{28}$.

Este fenômeno se tornou evidente com os participantes da pesquisa, em que até os que se apresentavam mais retraídos demonstraram maior integração, pegando os instrumentos para tocar, participando dos corais, escolhendo a música que possui maior afeto, sorrindo e levantando para dançar. Essas atitudes evidenciam a melhora na dimensão afetiva, em que os resultados estatísticos fortalecem esta argumentação. Os estudos anteriores corroboram esse resultado ${ }^{27-29}$.

Um estudo realizado em uma escola pública objetivou analisar a influência da música no comportamento dos alunos. De um grupo que sempre aparentava desinteresse, insatisfação, desmotivação e outros afetos negativos, após as intervenções musicais, revelaram afetos positivos, como satisfação, valorização e motivação, o que possibilitou o estabelecimento de uma forte relação entre os professores e alunos com base nesses afetos. Identificou-se também que a música permite a expressão do indivíduo, demonstrar seus conhecimentos e visão de mundo ${ }^{27}$.

Em outro estudo, também realizado com crianças e adolescentes, apresentando problemas emocionais e comportamentais, observouse uma melhora dos níveis de depressão e autoestima, além do aprimoramento das habilidades comunicativas e interativas. Esses efeitos, no entanto, foram visualizados apenas a curto prazo, levando a questionar de que modo a intervenção com musicoterapia pode ser delineada para favorecer sua eficácia, considerando as características do público-alvo e do contexto ${ }^{30}$. Embora os participantes e o contexto sejam distintos, observa-se que a intervenção musical apresentou resultados similares aos encontrados nesta pesquisa.

A música, como tecnologia de cuidado na enfermagem, está presente na Classificação de Intervenções de Enfermagem (Nursing Intervention Classification - NIC), apresentada como uma intervenção a ser utilizada de maneira complementar, criteriosa, buscando a restauração do bem-estar, mudança específica no comportamento, sentimentos e engajando o sujeito na percepção de seu próprio processo saúde-doença. No entanto, existem poucos estudos sobre a utilização da música como intervenção no cuidado, podendo estar relacionado ao escasso conhecimento dessa tecnologia como recurso terapêutico ${ }^{30,31}$.

As utilizações da música por enfermeiros, encontradas no NIC, são identificadas na maioria das esferas do ser humano.
Algumas das intervenções voltadas para o contexto da saúde mental são: estimulação cognitiva, promovendo a percepção e compreensão do ambiente por meio da música; distração, utilizando a música como foco proposital para manter a atenção longe de sensações indesejadas; terapia de recordações, podendo utilizar da música como elemento deflagrador, facilitando o prazer e qualidade de vida ${ }^{31}$.

Além da falta de estudos sobre o tema, esses profissionais acabam se centrando nas tecnologias mais avançadas e no tratamento medicamentoso, devido ao modelo biomédico impregnado na formação do profissional e nos ambientes hospitalares, ao bloqueio do enfermeiro em se aproximar de forma sensível do usuário com transtorno mental agudo e persistente e pela falta de busca por parte do profissional em se familiarizar com a utilização da música no cuidado ${ }^{32}$.

Independentemente de toda a evolução e expansão das PIC no Brasil, seu uso ainda é bastante limitado, sendo utilizadas quase que exclusivamente na Atenção Primária à Saúde, em que praticamente $80 \%$ desses recursos são oferecidos ${ }^{33}$. Isto se dá pelo fato de, nos níveis secundários e terciários, o modelo biomédico ainda estar fortemente enraizado na visão de cuidado dos profissionais. Apesar de este modelo trazer inquestionáveis avanços técnico-científicos, ele não contempla a totalidade do sujeito, ignorando toda a complexidade deste como ser biopsicossocial-espiritual-cultural ${ }^{34}$.

Outra caraterística das $\mathrm{PIC}$ no Brasil se refere à sua distribuição. Uma análise do território nacional permite observar que pessoas com alto nível de escolaridade, maiores de 50 anos de idade, do sexo feminino, com duas ou mais doenças crônicas e residentes na região Norte compõem o perfil predominante que faz uso de PIC. Em relação aos recursos mais utilizados, estão as plantas medicinais/fitoterapia, acupuntura e homeopatia. Essa análise indica a importância de democratizar as PIC, ampliando o acesso às demais parcelas da sociedade, bem como aos outros recursos terapêuticos que compõem a política brasileira ${ }^{35}$.

Essa visão biomédica, por parte dos profissionais de saúde, ocorre pela existência de hegemonias associadas às ideologias, práticas e ao complexo médico-hospitalar. Além do viés biologicista, esta hegemonia apresenta caráter mercantilista e corporativista, contendo práticas de propensão controladora, medicalizante e autoritária, o que se pode observar fortemente na atenção hospitalar psiquiátrica, no qual o modelo manicomial persiste ${ }^{36}$.

É a partir deste contexto que se observa a importância de transitar com as PIC para a atenção hospitalar. O uso dessas nesses ambientes constitui um caráter contra-hegemônico, ao centrar o cuidado no usuário com transtorno mental grave e persistente e, em seu contexto, integrar a prevenção e promoção da saúde, servir como intervenção à ideologia de medicalização, deslocá-lo para o protagonismo de seu cuidado, além de diversas outras afinidades que as práticas complementares compartilham com a atenção psicossocial ${ }^{36}$. Desse modo, a oferta da oficina musical participativa representou um recurso explorando sentidos socializadores e integrativos, em detrimento de ações ligadas à hegemonia biomédica. 
A utilização dessas práticas nesses setores, para além da atenção primária, permitirá que o foco não seja mais exclusivamente centrado na doença, com a visão fragmentada do usuário com transtorno mental agudo e persistente, mas que o cuidado seja integral, desconstruindo a prática manicomial, mudando este modelo para o modo psicossocia| ${ }^{33-36}$. Esse tensionamento positivo provocado pela oficina musical participativa no contexto da internação psiquiátrica tem evidente alinhamento teórico com pressupostos da Reforma Psiquiátrica. As PIC operacionalizadas nos serviços de saúde mental representam concretamente uma prática que converge para as dimensões técnico-assistencial, teórico-conceitual e sociocultural, daí sua potência para a efetivação do modo psicossocial no cuidado ${ }^{36,37}$.

Em um estudo norte-americano, comparou-se a terapia musical com a terapia de grupo padrão do hospital psiquiátrico em questão. Os resultados indicaram que o grupo que recebeu intervenção musical apresentou um aumento da qualidade de vida em comparação ao grupo padrão. O fato de os grupos de musicoterapia serem iniciados no âmbito hospitalar sem a necessidade de profissionais ou financiamento adicionais sugere que a criação de grupos de intervenção musical é viável no ambiente de internação psiquiátrica ${ }^{38}$.

A implementação da musicoterapia complementa as outras terapias oferecidas nesses ambientes, provendo uma variedade de propostas de cuidado que atendam ao interesse dos usuários assim como um meio complementar de expressão e exploração emocional ${ }^{38}$. Similarmente, os participantes desta pesquisa se apresentaram interessados na intervenção, buscando participar cada um da sua maneira, seja cantando, dançando, tocando um instrumento, batendo palmas e até rezando. Afirma-se que a música tornou o ambiente propício para que os participantes pudessem expressar suas emoções, proporcionando o exercício da autonomia, na seleção das músicas, do instrumento musical e se desejavam participar ou não.

No que se refere às características sociodemográficas da população do estudo, observa-se uma maioria de pessoas do sexo feminino, sem parceria, sem filhos e com até oito anos de escolaridade. Em relação às características clínicas, não houve ocorrências para consumo de álcool ou substâncias ilícitas. Identificaram-se diagnósticos de transtorno de pensamento e afetivo, ambos com tratamento medicamentoso, cujo predomínio foi para antipsicóticos, benzodiazepínicos e antidepressivos. Estudo da região sudeste indica predominância masculina, transtornos psicóticos e tempo médio de internação de 31 dias; dessas características, apenas sexo difere da amostra do presente estudo ${ }^{39}$.

O usuário com transtorno mental grave e persistente não deve ser entendido como um conjunto orgânico de processos fisiológicos, mas sim como um ser, com seus desejos, sonhos e emoções, que está inserido em um contexto histórico, social e cultural. Ele não é um objeto que deve ser manipulado, mas alguém capaz de atuar em seu próprio processo saúde-doença.

Nessa direcionalidade, o cuidado em saúde mental deve objetivar à promoção da autonomia e da emancipação do usuário por meio de recursos terapêuticos que pluralizem o cuidado e ampliem a clínica, ao mesmo tempo que provoquem mudanças no modelo de atenção à saúde e irrompam a persistência do modelo biomédico e da medicalização.

Para tanto, a intervenção musical é um recurso para o cuidado que auxilia o enfermeiro em seus planos e suas intervenções de cuidado para atuar de modo integral e integrado nas dimensões que constituem o sujeito. O emprego da terapia musical é uma ferramenta valiosa de amparo não farmacológico, contudo subvalorizada, para qualificar, a atenção os usuários com transtorno mental grave e persistente ${ }^{30,40}$.

Os enfermeiros são profissionais de saúde habilitados para estar sempre em relação com os usuários, fator que os tornam centrais em termos de gerenciamento e prestação dos cuidados mais efetivos. Os efeitos da musicoterapia e sua contribuição para o cuidado da enfermagem psiquiátrica e em saúde mental são fundamentais por suas qualidades integrativa, reabilitatória e socializadora, favorecendo a saúde dos usuários, a qualidade da assistência e a redução de custos ${ }^{41}$.

\section{CONCLUSÕES E IMPLICAÇÕES PARA A PRÁTICA}

Frente ao exposto, foi possível identificar as contribuições de uma intervenção musical participativa no campo do BES, mais precisamente nas dimensões dos afetos positivos e negativos, em usuários da unidade de internação psiquiátrica. Os efeitos da música para com este aspecto já vêm sendo discutidos e confirmados pela literatura científica, como se pode observar em diversos artigos.

No desfecho do BEP, os resultados não demonstraram significância estatística. Esse fato pode ser explicado pelo momento em que se encontravam os participantes, caracterizado por crise e labilidade emocional, elemento que atuou como um limitador do estudo. O comportamento emocional dos participantes durante as oficinas foi substancialmente favorável, escolhendo as músicas, auxiliando na execução das melodias e letras, situação que demonstra satisfação e sentimentos positivos que reverberaram no período da internação. Assim, mais estudos sobre a influência de intervenções musicais no BEP devem ser realizados, principalmente no contexto dos serviços de saúde mental.

A música como tecnologia leve pode ser facilmente utilizada pelos profissionais que estão na assistência, pois não demanda de todo um aparato de alta tecnologia e dificuldade, além do nítido custo-benefício para a instituição e/ou profissionais. Nesse estudo, a quantidade de oficinas pode ter sido um desafio para a composição da amostra de participantes, contudo a análise dos resultados permite inferir que poderiam ter sido realizadas de maneira independente, com resultados favoráveis já na primeira oferta. Vale ressaltar que a musicoterapia não deve ser uma intervenção imposta ao usuário com transtorno mental grave e persistente, mas ofertada respeitando o desejo e a predileção musical dos usuários. 
É de grande relevância que haja o estímulo dos profissionais da enfermagem para o uso desta tecnologia leve no cuidado, em especial na saúde mental, entre os dispositivos da RAPS. O modo biomédico atravessa a produção de cuidado em todos estes dispositivos, em que a busca pela medicalização do sofrimento é uma estratégia permanente e multifacetária. Este estímulo pode vir por meio da experimentação da potência de outros modos de se produzir saúde, aqui representado na oficina musical participativa; da sensibilização e conscientização de que o processo de adoecimento vai além da dicotomia saúdedoença, entre outros.

\section{CONTRIBUIÇÕES DOS AUTORES}

Desenho do estudo. Eduardo Gabriel Cassola. Márcia Caroline dos Santos. Bárbara Vukomanovic Molck. João Vitor Pereira da Silva. Thiago da Silva Domingos. Guilherme Correa Barbosa.

Aquisição de dados. Eduardo Gabriel Cassola. Márcia Caroline dos Santos. Bárbara Vukomanovic Molck. João Vitor Pereira da Silva. Thiago da Silva Domingos. Guilherme Correa Barbosa.

Análise de dados e interpretação dos resultados. Eduardo Gabriel Cassola. Márcia Caroline dos Santos. Bárbara Vukomanovic Molck. João Vitor Pereira da Silva. Thiago da Silva Domingos. Guilherme Correa Barbosa.

Redação e revisão crítica do manuscrito. Eduardo Gabriel Cassola. Márcia Caroline dos Santos. Bárbara Vukomanovic Molck. João Vitor Pereira da Silva. Thiago da Silva Domingos. Guilherme Correa Barbosa.

Aprovação da versão final do artigo. Eduardo Gabriel Cassola. Márcia Caroline dos Santos. Bárbara Vukomanovic Molck. João Vitor Pereira da Silva. Thiago da Silva Domingos. Guilherme Correa Barbosa.

Responsabilidade por todos os aspectos do conteúdo e a integridade do artigo publicado. Eduardo Gabriel Cassola. Márcia Caroline dos Santos. Bárbara Vukomanovic Molck. João Vitor Pereira da Silva. Thiago da Silva Domingos. Guilherme Correa Barbosa.

\section{EDITOR ASSOCIADO}

Antonio José Almeida Filho (1)

\section{EDITOR CIENTÍFICO}

Marcelle Miranda da Silva (D)

\section{REFERÊNCIAS}

1. World Health Organization. WHO global report on traditional and complementary medicine 2019 [Internet]. Geneva: WHO; 2019 [citado 2021 jun 4]. Disponível em: https://www.who.int/publications/i/ item/978924151536

2. Areias JC. A música, a saúde e o bem estar. Nascer Crescer [Internet]. 2016; [citado 2020 jul 5];25(1):[aprox.4 telas]. Disponível em: http://www.scielo. mec.pt/scielo.php?script=sci_arttext\&pid=S0872-07542016000100001

3. Zanello V, Souza G. Mais música, menos Haldol: uma experiência entre música, Phármakon e loucura. Mental [Internet]. 2009; [citado 2020 jul 6];7(13):[aprox.12 telas]. Disponível em: http://pepsic.bvsalud.org/ scielo.php?pid=S1679-44272009000200009\&script=sci_abstract

4. Wang S, Agius M. The use of music therapy in the treatment of mental illness and the enhancement of societal wellbeing. Psychiatr Danub. 2018 nov;30(7, Supl. 7):595-600. PMid:30439854

5. Santos TRMS, Cavalcante TB, Silva JJF. Music therapy in patients with disorders of consciousness: an integrative review. Cad Bras Ter Ocup. 2019 maio;27(4). http://dx.doi.org/10.4322/2526-8910.ctoar1909.

6. Weigsding JA, Barbosa CP. A influência da música no comportamento humano. Arquivos do MUDI [Internet]. 2018 nov; [citado 2020 jun 3];18(2):47-62. Disponível em: https://www.meloteca.com/wp-content/ uploads/2018/11/a-influencia-da-musica-no-comportamento-humano. pdf

7. Ramalho ADM, Ramalho JPG. A musicoterapia como recurso terapêutico para tratamento do paciente psiquiátrico. Enfermagem Brasil [Internet] 2017 ago; [citado 2020 jul 6];16(4):246-52. Disponível em: https:// portalatlanticaeditora.com.br/index.php/enfermagembrasil/article/ view/1263/2398

8. Siqueira MMM, Padovam VAR. Bases teóricas de bem-estar subjetivo, bem-estar psicológico e bem-estar no trabalho. Psicol, Teor Pesqui. 2008 jun;24(2):201-9. http://dx.doi.org/10.1590/S0102-37722008000200010.

9. Zanon C, Bastianello MR, Pacico JC, Hutz CS. Desenvolvimento e validação de uma escala de afetos positivos e negativos. Psico-USF. 2013 ago;18(2):193-201. http://dx.doi.org/10.1590/S1413-82712013000200003.

10. Carvalho HW, Andreoli SB, Lara DR, Patrick CJ, Quintana MI, Bressan RA et al. Structural validity and reliability of the Positive and Negative Affect Schedule (PANAS): evidence from a large Brazilian community sample. Rev Bras Psiquiatr. 2013 jun;35(2):169-72. http://dx.doi. org/10.1590/1516-4446-2012-0957. PMid:23904023.

11. Portaria n. 971/2006 (BR). Aprova a Política Nacional de Práticas Integrativas e Complementares (PNPIC) no Sistema Único de Saúde. Diário Oficial da União [periódico na internet], Brasília (DF), 4 maio 2006: Seção 1:20-5] [citado 19 jun 2017]. Disponível em: https://bvsms. saude.gov.br/bvs/saudelegis/gm/2006/prt0971_03_05_2006.html

12. Portarian. 145/2017 (BR). Altera procedimentos na Tabela de Procedimentos, Medicamentos, Órteses, Próteses e Materiais Especiais do SUS para atendimento na Atenção Básica. Diário Oficial da União [periódico na internet], Brasília (DF), 13 jan 2017: Seção 1: $32-4$ [citado 1 jun 2018]. Disponível em: http://pesquisa.in.gov.br/imprensa/jsp/visualiza/index. jsp? jornal=1\&pagina $=32 \&$ data $=13 / 01 / 2017$

13. Portaria n. 702/2018 (BR). Altera a Portaria de Consolidação no 2/GM/ MS, de 28 de setembro de 2017, para incluir novas práticas na Política Nacional de Práticas Integrativas e Complementares - PNPIC. Diário Oficial da União [periódico na internet], Brasília (DF), 21 mar 2018 Seção 1: $34-40$ [citado 1 jun 2018]. Disponível em: http://bvsms.saude. gov.br/bvs/saudelegis/gm/2018/prt0702_22_03_2018.html

14. Dacal MPO, Silva IS. Impactos das práticas integrativas e complementares na saúde de pacientes crônicos. Saúde Debate. 2018 set;42(118):72435. http://dx.doi.org/10.1590/0103-1104201811815.

15. Anjos AG, Montanhaur CD, Campos ÉB, Piovezana ALPD, Montalvão JS Neme CMB. Musicoterapia como estratégia de intervenção psicológica com crianças: uma revisão da literatura. Gerais Rev Interinst Psicol [Internet]. 2017 dez; [citado 2020 jul 6];10(2):228-38. Disponível em: http://pepsic. bvsalud.org/scielo.php?script=sci_arttext\&pid=S1983-82202017000200008

16. Lei n. 5.905 de 12 de julho de 1973 (BR). A resolução COFEN no $581 / 2018$ - alterada pela resolução COFEN no 625/2020, atualiza, no âmbito do Sistema Cofen/Conselhos Regionais de Enfermagem, os procedimentos para Registro de Títulos de Pós - Graduação Lato e Stricto Sensu concedido a Enfermeiros e aprova a lista das especialidades. Diário Oficial da União, Brasília (DF), 11 jul 2018.

17. Azevedo C, Moura CC, Corrêa HP, Mata LRF, Chaves ÉCL, Chianca TCM. Práticas integrativas e complementares no âmbito da enfermagem: aspectos legais e panorama acadêmico-assistencial. Esc Anna Nery. 2019 abr;23(2):e20180389. http://dx.doi.org/10.1590/2177-9465ean-2018-0389.

18. Guimarães NA, Borba LO, Larocca LM, Maftum MA. Tratamento em saúde mental no modelo manicomial (1960 a 2000): histórias narradas por profissionais de enfermagem. Texto Contexto Enferm. 2013;22(2):361 9. http://dx.doi.org/10.1590/S0104-07072013000200012. 
19. Cardoso L, Galera SAF. O cuidado em saúde mental na atualidade. Rev Esc Enferm USP. 2011 jun;45(3):687-91 . http://dx.doi.org/10.1590/ S0080-62342011000300020. PMid:21710076.

20. Sampieri RH, Collado CF, Lucio MPB. Metodologia de pesquisa. 5. ed. Porto Alegre: Penso; 2013.

21. Galinha IC, Pais-Ribeiro JL. Contribuição para o estudo da versão portuguesa da Positive and NegativeAffect Schedule (PANAS):II estudo psicométrico. Anal Psicol. 2005 abr;23(2):219-27. http://dx.doi. org/10.14417/ap.84.

22. Machado WL, Bandeira DR, Pawlowski J. Validação da Psychological Well-being Scale em uma amostra de estudantes universitários. Aval Psicol [Internet]. 2013 ago; [citado 2020 jul 6];12(2):263-72. Disponível em: http://pepsic.bvsalud.org/scielo.php?script=sci_arttext\&pid=S167704712013000200017\&lng=pt

23. Resolução n. 466/2012 (BR). Dispõe sobre diretrizes e normas regulamentadoras de pesquisas envolvendo seres humanos. Diário Oficial da União [periódico na internet], Brasília (DF), 12 dez 2012. [citado 1 jun 2018]. Disponível em: http://www.conselho.saude.gov. br/resolucoes/2012/Reso466.pdf

24. Miasso Al, Cassiani SHB, Pedrão LJ. Transtorno afetivo bipolar e ambivalência em relação à terapia medicamentosa: analisando as condições causais. Rev Esc Enferm USP. 2011 abr;45(2):433-41. http:// dx.doi.org/10.1590/S0080-62342011000200019. PMid:21655795.

25. Ferreira BC Jr, Barbosa MA, Barbosa IG, Hara C, Rocha FL. Alterações cognitivas na esquizofrenia: atualização. Rev Psiquiatr Rio Gd Sul. 2010;32(2). http://dx.doi.org/10.1590/S0101-81082010000200006.

26. Silva MJP. Comunicação tem remédio: a comunicação nas relações interpessoais em saúde. 8. ed. São Paulo: Edições Loyola; 2011.

27. Neves MAP, Souza VLT. Música e psicologia na escola: mobilizando afetos na classe de recuperação. Psicol Esc Educ. 2018 jan/abr;22(1):17-25. http://dx.doi.org/10.1590/2175-35392018019065.

28. Gebhardt S, Dammann I, Loescher K, Wehmeier PM, Vedder H, von Georgi R. The effects of music therapy on the interaction of the self and emotions: an interim analysis. Complement Ther Med. 2018 dez;41:616. http://dx.doi.org/10.1016/j.ctim.2018.08.014. PMid:30477866.

29. Porter S, McConnell T, McLaughlin K, Lynn F, Cardwell C, Braiden $\mathrm{H}-\mathrm{J}$ et al. Music therapy for children and adolescents with behavioura and emotional problems: a randomised controlled trial. J Child Psychol Psychiatry. 2017 maio;58(5):586-94. http://dx.doi.org/10.1111/jcpp.12656. PMid:27786359.

30. Franzoi MAH, Santos JLG, Backes VMS, Ramos FRS. Intervenção musical como estratégia de cuidado de enfermagem a crianças com transtorno do espectro do autismo em um centro de atenção psicossocial. Texto Contexto Enferm. 2016 Mar;25(1). http://dx.doi. org/10.1590/0104-070720160001020015.

31. Gloria MB, Howard KB, Joanne MD. Classificação das intervenções de enfermagem (NIC). 5. ed. Rio de Janeiro; 2010.

32. Bergold LB, Alvim NAT. A música terapêutica como uma tecnologia aplicada ao cuidado e ao ensino de enfermagem. Esc Anna Nery. 2009 set;13(3):537-42. http://dx.doi.org/10.1590/S1414-81452009000300012.

33. Amado DM, Rocha PRS, Ugarte OA, Ferraz CC, Lima MC, Carvalho FFB. Política Nacional de Práticas Integrativas e Complementares no Sistema Único de Saúde 10 anos: avanços e perspectivas. J Manag Prim Heal Care. 2018;8(2):290-308. http://dx.doi.org/10.14295/jmphc. v8i2.537.

34. Melo SCC, Santana RG, Santos DC, Alvim NAT. Práticas complementares de saúde e os desafios de sua aplicabilidade no hospital: visão de enfermeiros. Rev Bras Enferm. 2013 nov/dez;66(6):840-6. http://dx.doi. org/10.1590/S0034-71672013000600005. PMid:24488454.

35. Boing AC, Santiago PHR, Tesser CD, Furlan IL, Bertolfi AD, Boing AF Prevalence and associated factors with integrative and complementary practices use in Brazil. Complement Ther Clin Pract. 2019;37:1-5. http:// dx.doi.org/10.1016/j.ctcp.2019.07.009. PMid:31445361.

36. Tesser CD, Sousa IMC. Atenção Primária, Atenção Psicossocial, Práticas Integrativas e Complementares e suas afinidades eletivas. Saude Soc. 2012 jun;21(2):336-50. http://dx.doi.org/10.1590/S010412902012000200008.

37. Amarante $\mathrm{P}$, Nunes $\mathrm{MO}$. A reforma psiquiátrica no SUS e a luta por uma sociedade sem manicômios. Cien Saude Colet. 2018;23(6):206774. http://dx.doi.org/10.1590/1413-81232018236.07082018 PMid:29972514.

38. Deatrich KG, Prout MF, Boyer BA, Yoder SE. Effectiveness of group music therapy in a psychiatric hospital: a randomized pilot study of treatment outcome. Int J Group Psychother. 2016 jun;66(4):592-617. http://dx.doi.org/10.1080/00207284.2016.1190239.

39. Lara APM, Volpe FM. Evolução do perfil das internações psiquiátricas pelo Sistema Único de Saúde em Minas Gerais, Brasil, 2001-2013. Cien Saude Colet. 2019;24(2):659-68. http://dx.doi.org/10.1590/141381232018242.14652017. PMid:30726398.

40. Witusik A, Pietras T. Music therapy as a complementary form of therapy for mental disorders. Pol Merkur Lekarski. 2019;47(282):240-3. PMid:31945027.

41. CiğerciY, Kisacik OG, Özyürek P, Çevik C. Nursing music intervention: a systematic mapping study. Complement Ther Clin Pract. 2019 maio;35:10920. http://dx.doi.org/10.1016/j.ctcp.2019.02.007. PMid:31003646. 